\section{APPROACH TO}

McGill Journal of Medicine

\section{Ocular Trauma}

\section{Jobanpreet Dhillon ${ }^{1}$}

${ }^{1}$ Faculty of Medicine, McGill University, Montréal, Québec, Canada

\section{Correspondence}

Jobanpreet Dhillon

Email: jobanpreet.dhillon@mail.mcgill.ca

\section{Publication Date}

January 22, 2021

MJM 2021 (19) 6

https://doi.org/10.26443/mjm.v19i1.322

\section{के McGill \\ Journal of Medicine}

www.mjmmed.com

\section{c) (1) (2)}

This work is licensed under a Creative Commons BY-NC-SA 4.0 International License.

\begin{abstract}
Ocular trauma can be a common presentation in the emergency department. It is therefore important for a physician to be able to quickly recognize vision-threatening conditions and provide necessary medical management prior to consulting ophthalmology. This article describes the pertinent information that should be gathered during a focused ocular history in a patient with ocular injury, and also provides a systematic approach to evaluating ocular trauma. As an example, a case study of open globe injury is used to illustrate the appropriate pre-ophthalmologic management and common medical errors that must be avoided for a good prognosis. Additional ocular conditions such as traumatic hyphema, traumatic optic neuropathy, traumatic vitreous hemorrhage, orbital compartment syndrome, chemical burns, and eyelid lacerations are also described as differential diagnosis. Ultimately, the aim of this work is to provide medical students with a fundamental understanding in approaching ocular trauma in emergency clinics.
\end{abstract}

\section{- KEYWORDS}

Ocular trauma, Ocular chemical injury, Orbital compartment syndrome, Open globe injury, Hyphema, Vitreous hemorrhage, Retinal detachment

\section{1 | QUESTION}

A 36-year-old male presents to the emergency clinic with sudden onset of pain, redness, and bleeding from his left eye that started while operating a woodcutter machine three hours ago. He irrigated his eye at home with water and took Tylenol ${ }^{\circledR}$ but noted that the bleeding and pain did not stop. He also reports blurry vision and some sensitivity to light in his left eye. Upon initial inspection you observe a 'teardrop' shaped pupil, conjunctival redness, and three small woodchips protruding out of the corneal limbus and scleral region in the left eye. There is no hyphema, no proptosis, and no apparent deformity of the globe. His past medical and ocular history is unremarkable, and he is currently not taking any medication. He does not wear any glasses or con- 
tact lens. No eye protection was worn at the time of injury.

Visual tests

- Visual acuity (VA): 20/25 OD and 20/150 OS

- The text in the entries may be of any length.

- Visual fields by confrontation: normal

- Relative afferent pupillary defect (rAPD): inconclusive (patient did not cooperate)

- Ocular motility: Full range, discomfort with left eye

- Intraocular pressure (IOP): not measured

- Red reflex: visible, symmetrical

- Eye $\mathrm{pH}: 7.2$

What is the next best step in management of this patient prior to consulting ophthalmology?

A. Continue to irrigate with saline

B. Place an eye shield over the affected eye

C. Put tetracaine (anesthetic) eye drops to relieve pain

D. Carefully remove the protruding woodchips

E. Perform an orbital ultrasound to determine extent of injury

\section{2 | ANSWER}

B. The 'teardrop' pupil along with protruding foreign body raises suspicion of an open globe injury. The best course of action is to protect the eye with an eye shield and obtain urgent ophthalmology consultation. Removal of foreign body should be deferred to the ophthalmologist, and one should avoid placing any medication (e.g. tetracaine) or diagnostic eye drops (e.g. fluorescein) into the affected eye. Maneuvers that may increase intraocular pressure and risk extrusion of intraocular contents are contraindicated; therefore, eye irrigation, IOP measurements with tonometry, orbital ultrasound, and eyelid retraction should not be performed.

\section{3 | INITIAL APPROACH}

Evaluation of a patient with suspected ocular trauma begins by identifying and treating any life-threatening injuries. Once medically stable, the physician should obtain a focused ocular history to identify any visionthreatening conditions. (1) This includes information regarding the injury, such as:

1. Mechanism: high-velocity projectiles, blunt trauma, or chemical exposure

2. Timing: acute or chronic

3. Location: home, work, or motor vehicle accident

4. Symptoms: diplopia, photophobia, pain with eye movement, or facial numbness

5. Past ocular history: pre-injury visual acuity, cataract, glaucoma, or retinal detachment

Other elements to note on history include current medications, drug allergies, tetanus immunization status, and prior anesthesia complications. (1) Next, an organized approach should be utilized to rapidly assess imminent threats to vision (Figure 1). Careful inspection of the ocular and orbital anatomy, along with comprehensive visual examination provides further information on the extent of trauma. $(1,2)$

In the present case, the patient was suspected to have an open globe injury, which involves a full thickness break of the eye wall composed of the sclera (white outer layer of the eyeball) and the cornea (transparent part of the eye covering the iris and pupil). Caused by sharp or blunt trauma, patients suffering from open globe injury present with acute eye pain that may or may not be accompanied with reduced VA. (3) Inspection with penlight or slit lamp may reveal eccentric or 'teardrop' pupil (Figure 2a), extrusion of vitreous (Figure $2 b$ ), possible loss of globe contour, and deep or shallow anterior chamber depth depending on the type and position of injury. $(1,4)$ In this case, the patient presented with pain, bleeding, teardrop pupil, and protruding woodchips from the eye wall, suggesting a highvelocity penetration injury likely from the projectile debris when operating wood-cutting machinery without ocular protection.

Once an open globe injury is suspected, protecting the affected eye with an eye shield and consulting ophthalmology is the first step in management. Avoid fur- 


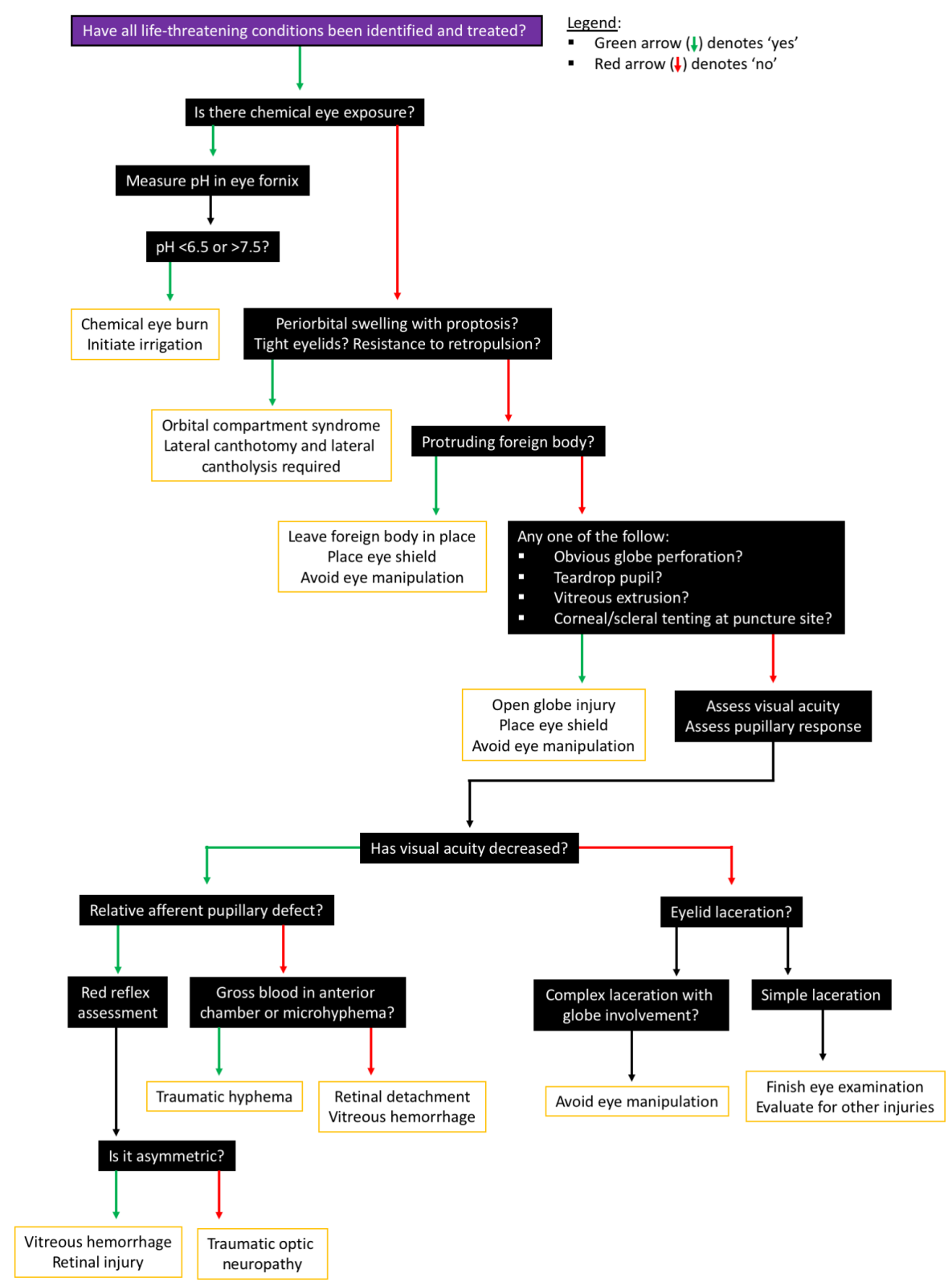

FIGURE 1 Organized approach for the assessment of ocular trauma in emergency department.

Adapted from: Approach to eye injuries in the emergency department, UpToDate ${ }^{\circledR}$ https://www.uptodate.com/contents/ approach-to-eye-injuries-in-the-emergency-department?csi=271f7410-cbe8407a-883d-1a032c7971cf\&source=contentShare 

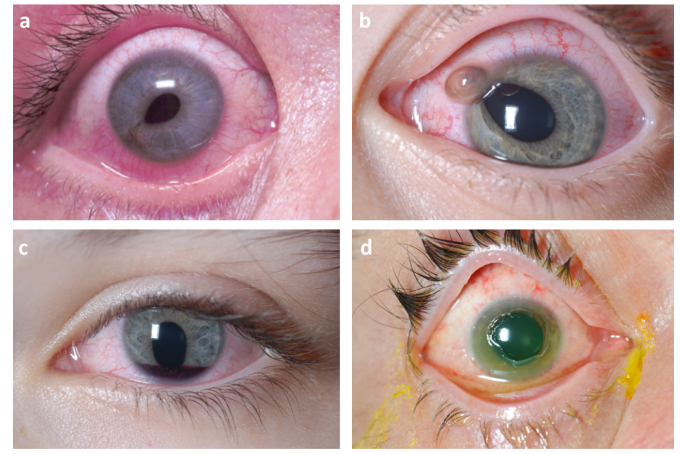

FIGURE 2 Presentation of the affected eye following ocular trauma. (a) Teardrop pupil pointing towards the location of corneal injury and suggestive of open globe injury. (b) Extrusion of intraocular content (iris prolapse) through an open globe defect. (c) Hyphema as noted by the accumulation of red blood cells in the anterior chamber. (d) Severe alkali chemical burn that resulted in a large corneal defect.

Images obtained from: Serrano, F., Stack, L. B., Thurman, R. J., Phillips, L., \& Self, W. H. Traumatic eye injuries: management principles for the prehospital setting. JEMS. 2013; 38(12): 56-62.

https://www.jems.com/gallery/80545/traumatic-eyeinjury-management-principles-for-the-prehospitalsetting/

ther examination that may increase IOP such as orbital ultrasound, eyelid retraction or IOP measurements with tonometry, as these maneuvers can extrude intraocular contents (Figure 2b). $(3,4)$ Do not attempt to remove the protruding foreign bodies and refrain from placing any medication (e.g. tetracaine) or diagnostic eye drops (e.g. fluorescein) into the affected eye. Verify patient's tetanus status. Treat any nausea/vomiting and pain with antiemetics (e.g. ondansetron) and analgesics. Broadspectrum antibiotics can be started to decrease the risk of endophthalmitis. (4) Imaging includes non-contrast orbital computer tomography (CT) to confirm diagnosis, determine the extent of foreign body penetration, and guide the treatment approach.

Several other conditions may occur in isolation or concomitantly with open globe injury following ocular trauma. These should be noted in the differential diag- nosis, and can include the following:

\section{1 | Traumatic Hyphema}

Hyphema is the accumulation of red blood cells in the anterior chamber that can be visualized in a sitting patient with a penlight or slit lamp (Figure 2c). (5) Hyphema should be suspected if the patient presents with decreased VA, pain with pupillary constriction to bright light, anisocoria, iridodialysis, and increased IOP. Hyphema can result from blunt or penetrating trauma that damages the iris or ciliary body vessels. It can be graded from zero (microhyphema) to four depending on the amount of blood present in the anterior chamber. $(5,6)$ Visual prognosis depends on the etiology, grade of hyphema, and ocular complications such as re-bleeding, optic atrophy, synechiae, and corneal blood staining. (7) Patients with sickle cell disease and bleeding disorders are at higher risk for poor outcomes; therefore, solubility testing or hemoglobin electrophoresis is recommended in susceptible population (e.g. African or Mediterranean descent or positive family history). (6)

Initial management includes protecting the eye with an eye shield and bed rest with the head of bed elevated to 30 degrees. (5) Diagnostic imaging with non-contrast orbital CT is considered when open globe injury, intraocular foreign body, or orbital fracture is suspected. If open globe injury is ruled out, IOP can be measured and topical pain medications (e.g. tetracaine, proparacaine) can be administered. Avoid the use of NSAIDs and aspirin for pain control as their platelet-inhibiting properties can increase risk of bleeding; use oral acetaminophen or oxycodone instead. (6) Antiemetic therapy can help control nausea/vomiting and the associated increase in IOP.

\section{2 | Eyelid lacerations}

It is not uncommon for ocular or facial trauma to be accompanied by eyelid injuries, especially if the etiology includes injury from high velocity projectiles. The eyelids play a crucial role in protecting the eye globe and maintaining tear film distribution and drainage. (8) If eyelid 
lacerations are present in a suspected open globe injury, avoid manipulation of eyelid and follow the open globe injury guidelines. Eyelid trauma that requires immediate consultation by ophthalmology includes laceration through full thickness of the lid or the lid margin, laceration with orbital fat prolapse, and laceration involving the tear drainage system. (9) Furthermore, any laceration in the medial one-third of the eyelid should be suspect for having a canalicular laceration and requires ophthalmology consultation. (9) Lid lacerations that do not involve the eyelid margin can be repaired with simple interrupted running sutures within 12-36 hours for good prognosis. In the event of animal bites, prophylactic antibiotics that cover anaerobes and aerobes should be initiated, and prophylaxis for rabies and tetanus may be considered.

\section{3 | Traumatic optic neuropathy}

While indirect traumatic optic neuropathy (TON) from blunt trauma is more common, it can also occur with a direct penetrating or lacerating trauma from high velocity projectiles. (10) Patients with TON can present with decreased VA, visual field defects, achromatopsia (red colour desaturation), and rAPD (present only in unilateral or asymmetric TON). Diagnostic evaluation requires urgent CT imaging of the optic canal and consultation with the ophthalmologist as treatment is determined by underlying etiology. (10)

\section{4 | Orbital compartment syndrome (OCS)}

As the orbit is a confined space, a rise in volume in this compartment can occur with intraorbital hemorrhage or soft tissue swelling following penetrating or blunt trauma. OCS occurs when the intraorbital pressure surpasses the arterial perfusion pressure of the optic nerve. (11) Patients present with acute onset of markedly decreased VA, diplopia, rAPD, ophthalmoplegia, proptosis, periocular edema, and evidence of increased intraorbital pressure such as tight eyelids and resistance to retropulsion. If it remains uncorrected, OCS can result in perma- nent vision loss within hours. As a true emergency, ophthalmology should be immediately consulted. If the IOP is found to be extremely high, emergent decompression of the orbit may be provided by lateral canthotomy and inferior cantholysis. $(11,12)$ Additional management involves bed rest with head elevation, pain control, and prevention of sudden increases in IOP through cough suppressants, stool softeners and antiemetics.

\section{4 | BEYOND THE INITIAL AP- PROACH}

Although the patient presented with open globe injury, in this case, a complete history may reveal other ocular trauma that warrant immediate treatment. For example, construction site hazards can include risk of chemical exposure and head trauma from falling debris. Therefore, other ocular conditions to probe on history include:

\section{1 | Ocular chemical injury}

The extent of damage caused by chemical burns depends on the type of agent, volume, and duration of exposure. (13) Alkaline agents cause severe injury as they lead to liquefactive necrosis, which allows for deep intraocular penetration (Figure 2d). In contrast, acids cause coagulative necrosis which protects the eye from deeper chemical penetrations. (13) Patients presenting with decreased VA, eye pain, conjunctival redness, blepharospasm (inability to open eyes), and photophobia should be suspected for ocular chemical injury. If a chemical burn is established, irrigation with isotonic saline should be started immediately before conducting further ocular evaluation. Irrigation should continue manually (or with a Morgan lens) with maximal exposure to conjunctiva and cornea until a pH between 7.0 and 7.4 is established (30-60 $\mathrm{min}$ ) by placing a litmus paper at the conjunctival fornix. $(14,15)$ Once a neutral $\mathrm{pH}$ is maintained, the affected eye should be assessed for corneal abrasions, foreign bodies, and globe rupture. 


\section{2 | Traumatic Vitreous Hemorrhage}

Vitreous humor is a clear, gel-like substance that occupies the space between the retina and the lens. (16) Traumatic vitreous hemorrhage occurs when blood leaks into the areas in and around the vitreous humor. (17) While this condition suggests retinal detachment or tear, it can also be observed in patients with subarachnoid or subdural hemorrhage due to head trauma. $(16,17)$ Patients may complain of acute painless visual loss, red hue to vision, and new onset of cobweb-like floaters. (16) Fundoscopy is used to evaluate the optic disc, retina and the surrounding vessels. Decrease in the red reflex can be noted on fundoscopy when blood is present in the aqueous or vitreous humor. $(16,17) \mathrm{Di}$ agnostic imaging includes CT of the head in cases of head trauma, and an ophthalmologist provides definitive treatment.

\section{5 | CONCLUSION}

Ocular trauma is a common presentation in the emergency department. A focused ocular history should include the mechanism, timing, and location of the injury, along with patient's symptoms and past ocular history. It is crucial to rule out open globe injuries prior to performing any eye manipulation procedures including orbital ultrasound, eyelid retraction, or IOP measurements with tonometry. Careful evaluation of the ocular and periocular structures should be followed by assessment of VA, rAPD, confrontational visual fields, red reflex, and IOP. Depending on the diagnosis, medical management could be initiated to reduce patient's pain, nausea/vomiting, and anxiety. Ultimately, it is important to familiarize oneself with early recognition and preophthalmologic management of vision threatening conditions.

\section{REFERENCES}

1. Conrad DR. Ocular Trauma: Principles and Practice, Ferenc Kuhn, Dante J. Pieramici. Thieme (2002). Canadian Journal of Ophthalmology. 2004;39(7):802.

2. Harlan JB, Jr., Pieramici DJ. Evaluation of patients with oc- ular trauma. Ophthalmol Clin North Am. 2002;15(2):153-161. https://doi.org/10.1016/s0896-1549(02)00006-8

3. Pieramici DJ, Sternberg P, Aaberg TM, et al. A System for Classifying Mechanical Injuries of the Eye (Globe). American Journal of Ophthalmology. 1997;123(6):820-831. https://doi.org/10.1016/s0002-9394(14)71132-8

4. Blair K, Alhadi SA, Czyz CN. Globe Rupture. In: StatPearls. Treasure Island (FL): StatPearls Publishing; 2020.

5. Wilson FM. Traumatic hyphema. Pathogenesis and management. Ophthalmology. 1980;87(9):910-919. https://doi.org/10.1016/s0161-6420(80)35144-0

6. Walton W, Von Hagen S, Grigorian R, Zarbin M. Management of Traumatic Hyphema. Survey of Ophthalmology. 2002;47(4):297334. https://doi.org/10.1016/S0039-6257(02)00317-X

7. Papaconstantinou D, Georgalas I, Kourtis N, et al. Contemporary aspects in the prognosis of traumatic hyphemas. Clin Ophthalmol. 2009;3:287-290. https://doi.org/10.2147/opth.s5399

8. Cochran ML, Czyz CN. Eyelid Laceration. In: StatPearls. Treasure Island (FL): StatPearls Publishing; 2020.

9. Brown DJ, Jaffe JE, Henson JK. Advanced Laceration Management. Emergency Medicine Clinics of North America. 2007;25(1):83-99. https://doi.org/10.1016/j.emc.2006.11.001

10. Jang SY. Traumatic Optic Neuropathy. Korean J Neurotrauma. 2018;14(1):1-5. https://doi.org/10.13004/kjnt.2018.14.1.1

11. Roth FS, Koshy JC, Goldberg JS, Soparkar CNS. Pearls of orbital trauma management. Semin Plast Surg. 2010;24(4):398-410. https://doi.org/10.1055/s-0030-1269769

12. Lima V, Burt B, Leibovitch I, Prabhakaran V, Goldberg RA, Selva D. Orbital compartment syndrome: the ophthalmic surgical emergency. Surv Ophthalmol. 2009;54(4):441-449. https://doi.org/10.1016/j.survophthal.2009.04.005

13. Singh P, Tyagi M, Kumar Y, Gupta KK, Sharma PD. Ocular chemical injuries and their management. Oman J Ophthalmol. 2013;6(2):83-86. https://doi.org/10.4103/0974-620X.116624

14. Kuckelkorn R, Schrage N, Keller G, Redbrake C. Emergency treatment of chemical and thermal eye burns. Acta Ophthalmologica Scandinavica. 2002;80(1):4-10. https://doi.org/10.1034/j.1600-0420.2002.800102.x

15. Eslani M, Baradaran-Rafii A, Movahedan A, Djalilian AR. The ocular surface chemical burns. J Ophthalmol. 2014;2014:196827196827. https://doi.org/10.1155/2014/196827

16. Jena S, Tripathy K. Vitreous Hemorrhage. In: StatPearls. Treasure Island (FL): StatPearls Publishing; 2020.

17. Spraul CW, Grossniklaus HE. Vitreous Hemorrhage. Surv Ophthalmol. 1997;42(1):3-39. https://doi.org/10.1016/s00396257(97)84041-6 